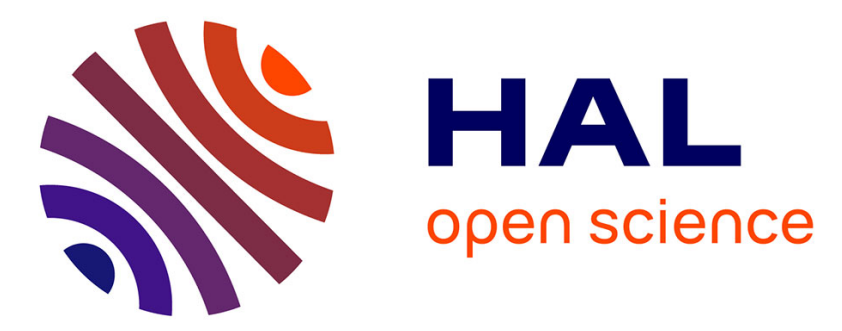

\title{
A Pluralist Framework to Address Challenges to the Modern Synthesis in Evolutionary Theory \\ Philippe Huneman
}

\section{To cite this version:}

Philippe Huneman. A Pluralist Framework to Address Challenges to the Modern Synthesis in Evolutionary Theory. Biological Theory, 2014, 9 (2), pp.163-177. 10.1007/s13752-014-0174-y . hal01968510

\section{HAL Id: hal-01968510 https://hal.science/hal-01968510}

Submitted on 10 Jan 2019

HAL is a multi-disciplinary open access archive for the deposit and dissemination of scientific research documents, whether they are published or not. The documents may come from teaching and research institutions in France or abroad, or from public or private research centers.
L'archive ouverte pluridisciplinaire HAL, est destinée au dépôt et à la diffusion de documents scientifiques de niveau recherche, publiés ou non, émanant des établissements d'enseignement et de recherche français ou étrangers, des laboratoires publics ou privés. 


\section{A Pluralist Framework to Address}

Challenges to the Modern Synthesis in Evolutionary Theory

\section{Philippe Huneman}

\section{Biological Theory}

ISSN 1555-5542

Biol Theory

DOI 10.1007/s13752-014-0174-y

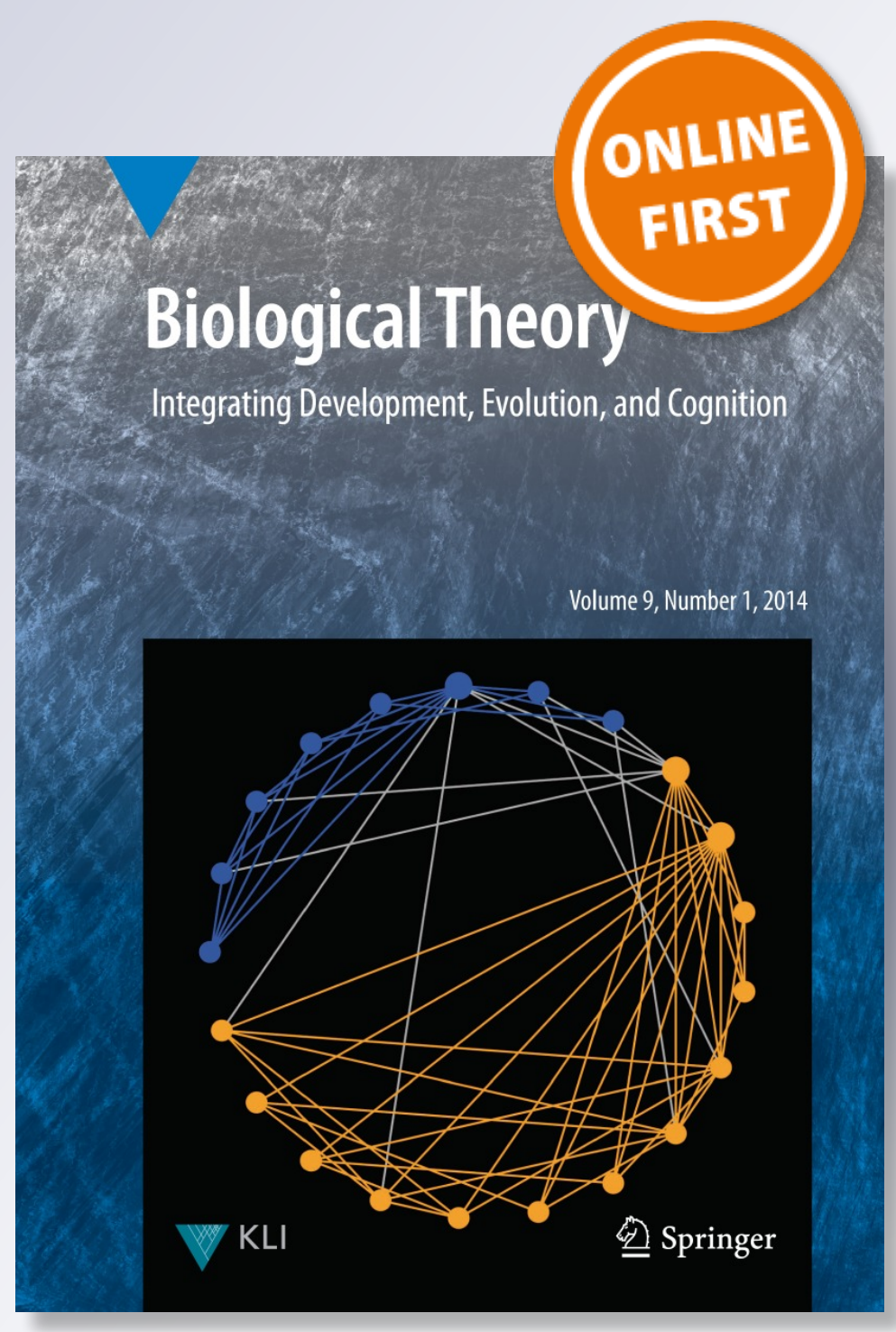

望 Springer 
Your article is protected by copyright and all rights are held exclusively by Konrad Lorenz Institute for Evolution and Cognition Research. This e-offprint is for personal use only and shall not be self-archived in electronic repositories. If you wish to self-archive your article, please use the accepted manuscript version for posting on your own website. You may further deposit the accepted manuscript version in any repository, provided it is only made publicly available 12 months after official publication or later and provided acknowledgement is given to the original source of publication and a link is inserted to the published article on Springer's website. The link must be accompanied by the following text: "The final publication is available at link.springer.com". 


\title{
A Pluralist Framework to Address Challenges to the Modern Synthesis in Evolutionary Theory
}

\author{
Philippe Huneman
}

Received: 8 May 2013/Accepted: 4 March 2014

(C) Konrad Lorenz Institute for Evolution and Cognition Research 2014

\begin{abstract}
This paper uses formal Darwinism as elaborated by Alan Grafen to articulate an explanatory pluralism that casts light upon two strands of controversies running across evolutionary biology, viz., the place of organisms versus genes, and the role of adaptation. Formal Darwinism shows that natural selection can be viewed either physicsstyle, as a dynamics of alleles, or in the style of economics as an optimizing process. After presenting such pluralism, I argue first that whereas population genetics does not support optimization, optimality can still be taken as a default hypothesis when modeling evolutionary processes; and second, that organisms have an explanatory role in evolutionary theory, since they are involved in the economic perspective of optimization. Finally, in order to ask whether the Modern Synthesis can indeed provide a theory of organisms, I apply a Kantian-inspired theoretical view of organisms (underlying much developmental modeling), according to which they are both designed entities and subjects of intrinsic circular processes involving the whole organism and its parts. I first show that the design aspect is accountable for in terms of the Modern Synthesis understood in the formal Darwinism framework. I then question whether the latter aspect of organisms can also be ultimately captured in the same framework, and to this purpose devise an empirical test relying on an assessment of the relative weight of genetic elements in developmental and functional gene regulatory networks.
\end{abstract}

\footnotetext{
P. Huneman $(\square)$

Institut d'Histoire et de Philosophie des Sciences et des Techniques (IHPST), CNRS/Université Paris I Sorbonne, Paris, France

e-mail: philippe.huneman@gmail.com
}

Keywords Adaptation - Formal Darwinism · Modern Synthesis - Natural selection - Optimization · Organism . Population genetics

It is commonly thought that Darwin provided a naturalistic explanation for the fact of adaptation and its pervasiveness in the organic world. Natural selection, by scrutinizing small variations and retaining the ones that increase reproductive chances, adapts organisms to their environment. Traits are there because they represent good answers to environmental demands, or what we can call solutions to design problems. ${ }^{1}$ Natural selection defines adaptations, since to be an adaptation for a trait means to result from selection (Brandon 1990), and specific selective pressures explain why adaptations are there. ${ }^{2}$ It is therefore tempting to see evolution by natural selection as a process that shapes organisms in order to maximize their fit to the environment. ${ }^{3}$ Natural selection and optimization thus go hand in hand. Natural selection in a sense replaced the

\footnotetext{
1 The idea that organisms solve design problems has been criticized by Lewontin (1978), among others. For example, there is an issue with what metrics to use to compare various solutions, not to mention the very idea of defining a "design problem." This article will deal extensively with the idea of design, and some of the issues raised by provisionally using the phrase "design problem" will be tackled along the way.

2 Niche construction theorists (see in particular Odling-Smee et al. 2003) contend that niche construction is an alternative pathway to adaptation; but this claim is controversial, and we will not consider it here.

3 The word "fitness," before being used by evolutionary biologists as a technical term, had a long history; it was extensively considered by natural theology, where it meant a sort of adjustment between organisms and their environment due to a divine intelligence, and attesting to it. The principle of natural selection (PNS) connects the fit between organisms and environment (often called "adaptedness") to the chances of differential reproduction; see Brandon (1990).
} 
divine architect; when Williams (1992) defined the criteria of adaptation for evolutionists by lengthily citing Paley, he exemplified such a trend.

Behavioral ecologists in particular are very much concerned with such a view. They often understand actual traits by comparing them to what would be the result of an optimizing computation by organisms of the trait value in the face of environmental demands. In this regard, there is a striking analogy between the optimizing behavior of economic agents that strive to choose the best action in order to maximize their utility, and organisms under natural selection acquiring seemingly optimal phenotypes because natural selection selects the ones with the highest fitness.

However, the modern understanding of natural selection that we call the Modern Synthesis (MS) traces back to population genetics, which unraveled the processes of selection as an aggregated dynamics of allele frequencies. In this perspective, evolutionary theory is much closer to statistical mechanics (as made famous by Fisher's analogy in his Genetic Theory of Natural Selection, 1930) than to economics. This point of view expels organisms from the core concepts of evolutionary theory. And it is this disregard that has been the focus of much criticism of the MS in the last two decades (Bateson 2005), coming especially from evolutionary developmental biology (EvoDevo) (Gilbert et al. 1996), even if the criticisms from various EvoDevo theorists (West-Eberhard 2003; Gilbert and Epel 2009) and some authors from other disciplines (Oyama 1985; Griffiths and Gray 1994; Griesemer 2000; OdlingSmee et al. 2003; Bateson 2005) are of a varied nature (Huneman 2010). As Walsh (2010) has suggested, many of the challenges to the MS can be understood in the following way: population geneticists such as Fisher and Wright, and then the molecular biologists discovering the substrate of inheritance after 1953, built a successful "suborganismic" biology. But recent advances in developmental theory, ecology of niche construction, and in our understanding of phenotypic plasticity (West-Eberhard 2003) call for an organismal biology.

The view that organisms are under optimization in such a way that optimality reasoning may ground our understanding of biological traits and structures, because natural selection is the main cause of the main traits, is equally controversial. This is known as the adaptationism problem. When it comes to the role of organisms, this problem is often conflated with issues about the role of organisms versus genes; but the two concerns are orthogonal. These two issues define the space of controversies about the MS. The two axes of this space are natural selection as optimization, and the role of genes versus organisms. Given that each axis determines two possible positions, there will be four poles in this theoretical space, viz., accepting that natural selection is the main agency (which means that there is a trend toward optimization that is explanatory in biology) or denying this; and accepting that evolution is mostly genetic, or not (and then, involves a causal role of organisms, for example). Figure 1 charts this space.

Certainly, philosophers of biology appreciate the clearcut contrast between Dawkins, holding both adaptationist and gene-centrist views, and Gould, who contested both the primordial role of genes and the fact that natural selection is the main agency of evolution. Actually, the orthodox view of MS could be summarized by this saying of Huxley: "Natural selection, acting on the heritable variation provided by the mutations and recombination of a Mendelian genetic constitution, is the main agency of biological evolution"- $a$ view that is obviously committed to two claims, one about natural selection and the other about genes. ${ }^{4}$ But from the beginnings of the MS to the present we can find instances of all combinations: some, such as famously Mayr's, would accept the claim that natural selection is the "main agency," but not the idea that evolution is a change in gene frequencies. ${ }^{5}$ Others would precisely accept the centrality of genes but not optimization. Thus Lynch (2007) ironically holds that "nothing in evolutionary biology makes sense except in the light of population genetics," but does not ascribe a prominent role to optimization since his own models show that drift is one of the main agents of the architecture of the metazoan genome. Extreme positions are exceptions, but they are useful to settle the borders of the space of controversies.

How can one understand this double issue of organisms/ genes and the place of adaptation? Is there a kind of pluralism that makes sense of coexisting views of evolution by natural selection? I will ask this question using the recent framework provided by Grafen $(2002,2006,2007)$ under the name of formal Darwinism (FD henceforth). I first present FD as a pluralism of economics and physics schemes. On this basis, I next specify the articulation between selection and optimization, and its consequences for the issue of adaptationism. I will then address the challenges presented to MS as a gene-centered biology by those who think that organisms should be given a crucial explanatory role. Based on FD, I will show that some equivalences hold between genetic and organismic viewpoints, and that these equivalences allow MS to rebut many of these challenges. I will finally indicate what should be the dimensions of organisms that may be wholly left aside from the MS, and where possibly empirical evidence

\footnotetext{
${ }^{4}$ Letter to Mayr, expressing what he expects from the contributors to his Evolution as a Process.

5 "Evolution is not a change in gene frequencies, as is claimed so often, but the maintenance (or improvement) of adaptedness and the origin of diversity. Changes in gene frequency are a result of such evolution, not its cause" (Mayr 1997, p. 2093).
} 
Fig. 1 Sketch of the space of controversies in evolutionary theory

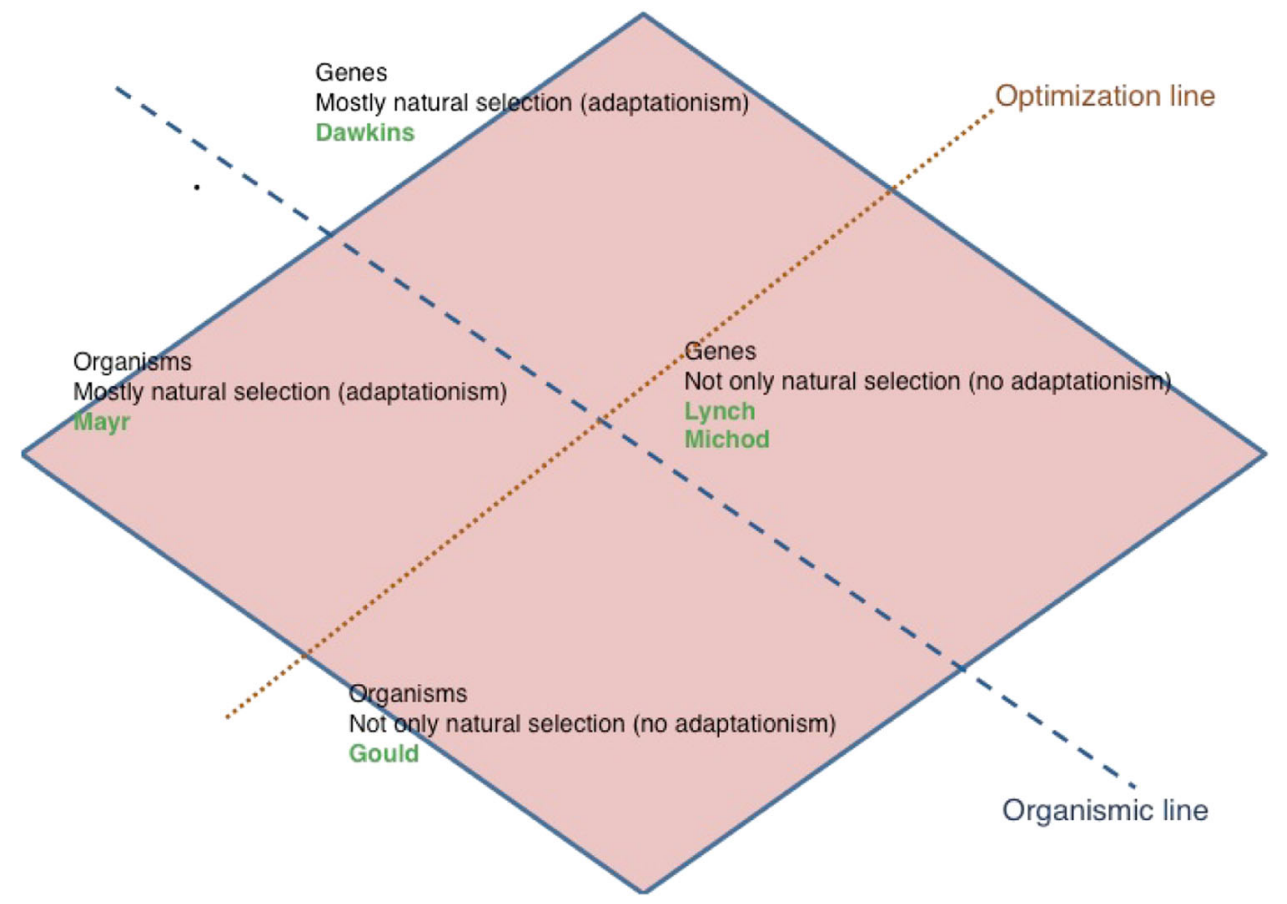

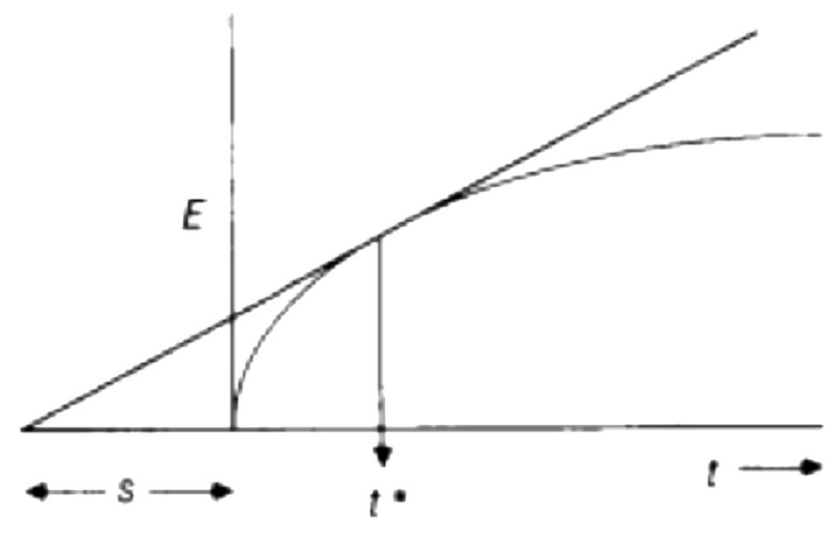

Fig. 2 Optimal foraging time $t^{*}$ determined as extremum of the derived function of $E=\mathrm{f}(t)$

should be searched to decide whether or not the explanatory project of understanding evolution should include such a dimension.

\section{Alleles and Strategies: Formal Darwinism as a Physics/ Economics Pluralism}

Optimality approaches embody the spontaneous vision of selection as adjusting organisms to their environments, hence optimizing the traits, whereas the population genetics approach conceives of selection as the statistical process of differential replication of alleles according to relative fitness values. For behavioral ecologists, a way to explain a trait consists of determining the function $w=\mathrm{f}(T)$, which relates fitness $w$ to the value of a trait $T$. One can here take a proxy $E$ for fitness, such as energy intake, metabolism rate, or other variables. The maximum for $w$ (or $E$, or the derived function of $E$ ) indicates what natural selection would select, and if the trait displays this value, it can be said that natural selection is the cause (Fig. 2). When natural selection is frequency-dependent, optimality methods are replaced by evolutionarily stable strategies, because there cannot be a fixed optimal value.

Whereas behavioral ecologists take for granted that selection maximizes fitness, population geneticists have pointed out many instances where genetic architecture prevents selection to maximize fitness. ${ }^{6}$ From the viewpoint of MS, a basis for optimization claims could be found in Fisher's fundamental theorem of natural selection (FTNS), which states that the increase in mean fitness in populations equals additive genetic variance, i.e., is always positive; thus fitness is maximized. On this simple reading, ${ }^{7}$ Fisher's theorem provides a first attempt to ground the common Darwinian intuition of optimization by selection. But Moran (1964) has shown with the help of population genetics models that the conclusion of the FTNS is not

\footnotetext{
6 "Most biologists who study adaptiveness in the field hold the view, following Darwin (1859), that natural selection leads organisms to act as if they are maximizing their fitness. This view is not supported by the current state of population genetics" (Grafen 2002, p. 75).

7 Notice that there is now a controversy about what is the exact meaning of FTNS, and some authors in the 1990s (Edwards 1994) convincingly established that a better reading does not claim a constant maximization of fitness in populations (see below).
} 
supported. The mathematical genetic theory of selection would prefer to state that optimization is not the norm, since many genetic settings prevent populations from reaching fitness optima. A simple example is the frequency dependence of social strategies: the aggressive strategy (hawk) has a higher fitness than the flight strategy (dove); however, if hawks increase in frequency, the advantage of being a hawk decreases relatively to being a dove (because hawks fight hawks and have chances to lose) (MaynardSmith 1982). Another example is heterozygote superiority: if the heterozygote genotype has a higher fitness than the two homozygotes, selection will increase the frequency of this genotype. However, it is impossible for it to dominate the population since homozygotes will be produced at each generation by recombination; therefore, selection does not maximize fitness. These two cases very generally are based on the same situation: the contextual dependency of the fitness of alleles, whether one considers the social (other strategies) or the genomic (other alleles) environment.

The general idea of Grafen's proof of equivalence is to show that with each population genetics model, given that it satisfies the Price equation — an a priori, mathematical truthone can associate an "optimization program," as another way of describing the system. The Price equation is written as $\Delta p_{\text {mean }}=\operatorname{Cov}_{\mathrm{i}}\left[p_{\mathrm{i}}, v_{\mathrm{i}}\right.$ (ignoring transmission biases) $]$, where $p$ is the phenotypic value and $v$ the fitness. It states intergeneration change in phenotypic value as a covariance between phenotypic value and the fitness difference. As an expression of gene dynamics, the Price equation is chosen for its generality, because it does not specify the relationship between phenotype and genotype. More precisely, the Price equation used here holds with $p$ being the heritable part of the phenotype, which can be equated for many purposes with the additive genetic value; Grafen calls this variable the $p$-score. The idea of the demonstration is then to consider phenotypes as various strategies, to construct an optimizing function for choosing among strategies, and show that this is logically equivalent to the Price equation.

Before making this more explicit, I will reject one possible objection. Some (Sober 1998) have argued that the analogy of choice and decision theory is not accurate in evolutionary biology because it yields false predictions concerning the evolution of cooperation. In this case, however, the formalism is deduced from the Price equation, and is not something already extant that one would apply externally to evolution. Grafen shows indeed that an optimization program can be written on the basis of the Price equation, a program whose natural interpretation consists of viewing phenotypes as strategies. Therefore FD is rather immune to general objections about decision theory in biology, even if such criticisms were right.

The inspiration of the formal derivation of the equivalence lies in the ability to express the whole population, considered in the Price equation, as one or several single "implicit decision makers" choosing between strategies, which can be considered as an ideal organism picking up its phenotypes. ${ }^{8}$ What has been established by Grafen as the basis of the population genetics / optimization scheme equivalence is that the fixation of the mean $p$-score predicted by the Price equation is then equivalent to the choice of a specific strategy $x^{*}$ within a strategy set $\mathrm{X}, x^{*}$ maximizing an optimization function $g$ that can for now be energy intake, offspring number, or any relevant value (think of all the proxies for fitness, such as energy intake, heat, etc., used in behavioral ecology), i.e., causally related to fitness. The most complete function $g$ is a function of environmental uncertainty $\gamma$ and available cues $s$; it defines what Grafen (2002) calls an "optimization program."

The crucial argument consists in showing that an isomorphism holds between the Price equation and the optimization program. To begin with, there are some considerations about population genetics. Grafen first makes the "strategic equivalence assumption" that all genes on average face the same environment-there is no privileged gene-environment association. This assumption is not realistic, but it aims at capturing the essential properties of selective dynamics before considering real cases. Like the simplest Newtonian models in dynamics it is a simplification, abstracting away from many real conditions (spatial heterogeneities in density, etc.). The framework of population genetics also includes various levels of uncertainty and various ploidy levels.

From here on one can build the model for the optimization framework and its $g$ function on the basis of parameters from population genetics. The arguments of the function are the strategies $x$, which correspond to phenotypes, and are possibly chosen by the "implicit decision maker" facing its environment. The construal of such $g$ function allows equivalences between the variables describing selection according to population genetics and optimization theory. Uncertainty is something that both theories should take into account and reflect in the equations. The population's environment can be represented by the cues according to which the decision maker will proceed. And the distinction between phenotype and realized phenotype - if one considers plasticity, bet-hedging, etc.is the difference between a conditional strategy and each of the actions done. Other correspondences are listed in Table 1.

Then the proof of the isomorphism between dynamics of gene frequencies and optimization requires understanding how statements holding in the former map onto statements holding in the latter. The process of fixing a $p$-score,

\footnotetext{
8 There are important formal constraints here if we want to carry on this reasoning, but they can be set aside for the present argument.
} 
Table 1 Correspondences between variables in population genetics and optimization (after Grafen 2002)

\begin{tabular}{|c|c|c|c|}
\hline \multicolumn{2}{|l|}{ Population genetics } & \multicolumn{2}{|c|}{ Optimization theory } \\
\hline Meaning & Notation & Notation & Meaning \\
\hline $\begin{array}{l}\text { Population measure } \\
\text { space }\end{array}$ & $(I, \underline{I}, \mu)$ & (none) & $\begin{array}{l}\text { Implicit decision- } \\
\text { taker }\end{array}$ \\
\hline General uncertainty & $\omega$ & $\gamma$ & General uncertainty \\
\hline And its measure space & $(\Omega, \underline{\Omega}$ & $(\Gamma, \underline{\Gamma}, n)$ & $\begin{array}{l}\text { And its measure } \\
\text { space }\end{array}$ \\
\hline Local environment & $r_{k}^{\omega}$ & $s=\sigma^{\gamma}$ & Cue \\
\hline Belongs to the set & $R$ & $S$ & Belongs to the set \\
\hline Phenotype & $a_{k}$ & $x$ & Strategy \\
\hline Realized phenotype & $a_{k}\left(r_{k}^{\omega}\right)$ & $X\left(\sigma^{\gamma}\right)$ & Action \\
\hline Belongs to the set & $A$ & $U_{s} X_{s}$ & Belongs to the set \\
\hline Target of selection & $v_{k}^{\omega}$ & $g$ & Maximand \\
\hline
\end{tabular}

described by the Price equation in population genetics, should correspond to the choice of a strategy by the "implicit decision maker" modeled in the optimization theory framework. If we get this, then, crucially, the dynamics of gene frequencies will involve a formula for the optimization program solved by what could be considered as an ideal organism choosing its strategies; and reciprocally, the optimization program solved by organisms can be written in terms of population genetics. ${ }^{9}$

Finally, we get such equivalence because of the fact (established by Grafen) that fixation of alleles according to the Price equation indeed yields ipso facto the maximization of the $g$ function $\left(g(x)=g^{*}\right)$. The reason is that when the function reaches its maximum $g^{*}$, then, given the construction of $g$, there is no variation in fitness ( $\mathrm{d} g$ / $\mathrm{d} x=0$ ). ${ }^{10}$ If there is no variation in fitness (no fitness differences), then, given the covariance between fitness and phenotypic value stated by the Price equation, the intergenerational difference in mean phenotypic value becomes null $\left(\Delta p_{\text {mean }}=0\right)$, hence the $p$-score fixed by selection according to the population dynamics is reached in the

\footnotetext{
9 This argument requires a caveat. There should be no intragenomic selection, which is part of the clauses Grafen assumes in order to build FD equivalence. However, this caveat does not alter the meaning of such equivalence. If there are organisms, then suborganismic selection has been somehow superseded by selection apparently taking place at the level of organisms (Grossberg and Strathmann 2007). But the entire FD project assumes that there are organisms, since it aims at understanding the relation between organismal and genetic explanations. Therefore, the no-intragenomic-selection assumption goes along with the very question of understanding such a relationship, and is not an objection to its answer as FD provides it. Understanding for which evolutionary reasons this holds is yet another empirical issue.

${ }^{10}$ To simplify the matter here, I assume that $g$ is a continuous function. This is oversimplifying but is supposed in order to verbally capture the logic.
}

population. In other words, due to the construction of the $g$ function, the implicit decision maker chooses as maximizing exactly a strategy that corresponds to the phenotypic values reached by selection in a population according to the Price equation.

Then comes the last step in this line of reasoning (Grafen 2006): in the first formulation $g$ could be defined within a range of possible fitness-related terms, but when one has to determine what it should be in cases where actions affect the fitness of other individuals (i.e., when there are social interactions), which were first excluded by the model, another abstract reasoning makes inclusive fitness the only possible maximand. Finally, population genetics formally corresponds to a view of individual organisms as maximizing agents, which are choosing their phenotypes in order to maximize their inclusive fitness.

\section{Selection and Optimization: Confronting Adaptationism in a Pluralist Framework}

The fundamental correspondence between fitness differences in population genetics and variation in the domain of the $g$ function, constitutive of the isomorphism that FD explains, can lead to four statements, which capture the various correspondences between optimization framework and population genetics, and therefore between an economic and a physical understanding of selection. That the optimization program is solved, or suboptimally solved, can indeed be immediately translated into statements about the scope and potentialities of selection, and vice versa. Therefore, the isomorphism can be expressed through four clauses, defining four cases. Regarding optimization, the achievement of optimization requires that selection have no more scope; conversely, if no more selection is possible, optimization is reached. As to suboptimality, there is a reciprocal link between a set of maximizing agents having reached the same suboptimal value and a subset of a gene pool such that no selection can happen because of the nullification of fitness differences, even if possible selection could happen if variations were introduced. Here, in Grafen's words, there is "no scope" for selection, but "potential" for it. This can easily be grasped with a reference to Wrightian fitness landscapes: when a population is stuck on a local optimum peak, only drift or migration can move it by taking it away from this peak; hence there is no "scope" for selection, since it is a peak, but still a "potential" since it can be shifted away from this peak. So, it is important to grasp that the formal underpinnings of selection as an optimizing process also allow for cases where optimal phenotypes are not reached. This will enable us to address the issue of adaptationism. 
Adaptation is a property of phenotypic traits. Notice that FD started with the Price equation because it assumes nothing regarding the genotype-phenotype map and genetic makeup. In this framework, we construct an optimization framework that basically views evolution by natural selection as a program "run" by organisms. This means that no knowledge of the genetic makeup is necessary to assume optimality as default, and therefore evolutionary biologists are entitled to ascribe traits to the action of selection even in the ignorance of genetic details. In this sense we here have a justification of the main assumption of behavioral ecology, and in general, of any study of evolution that does not delve into the genetic makeup of systems. This is crucial, because many phenotypes are in fact underpinned by hundreds of different alleles (height being a good example) unknown to biologists. Grafen (1984) called "phenotypic gambit" the general assumptions made by biologists studying phenotypic evolution, which is that in the long run allelic frequencies will tend to a combination underpinning the phenotype that will be reached by natural selection as predicted by the study of the environmental context. The isomorphism defined by FD, using the Price equation-which is neutral regarding genetic assumptions-thereby provides grounds for accepting this gambit. ${ }^{11}$

More precisely, biologists would often assume that the genetic values add up, and that in general all alleles make a small contribution to the phenotype. This was one of the common assumptions made by the population geneticists of the MS (Orr and Coyne 1992). The phenotypic gambit in these cases was quite likely to hold because small contributions adding up would gradually cohere into an ensemble result, in which none of them is prominent, and which matches some constraints imposed at the phenotypic level. The process would compare in some ways to statistical mechanics, where small elements of comparable individual effects add up into a predictable result (e.g., temperature or entropy) at the level of an ensemble. However, it might be that in some cases the contribution of these very different alleles is not of comparable magnitude (Marshall et al. 1999). Yet for the above-mentioned reasons the FD isomorphism still holds, which means that the phenotypic gambit is still justified, and that the methodology of considering phenotypes in themselves as answering

\footnotetext{
11 Yakubu (2013) criticizes the tradition of evolutionary investigations of cooperation and altruism on the basis that their models would presuppose genes for unconditional altruism or selfishness, instead of plastic phenotypes conditionally switching to altruism or selfishness. This latter situation-rather than unconditional genes-is, as he argues, widely attested by empirical findings. The "phenotypic gambit," however, is neutral regarding which case obtains, since, due to its justification, it is neutral regarding the genetic makeup of phenotypes.
}

environmental challenges via natural selection, notwithstanding their genetic bases, is still sound.

To this extent, the pluralistic framework provided by FD clarifies the debates on adaptationism. A first level of answer is just this phenotypic gambit, namely the methodological assumption that even though evolution is driven by the change in allelic frequencies, because it is the heritable part of the phenotype that only allows for persistent changes in the phenotypes, one can still focus on the phenotypic traits in order to understand what had been their evolution in the face of environmental context. The fact that the dynamics of allelic frequencies maps onto a process of maximizing a function whose arguments are phenotypic values justifies that one can model the evolution of traits by considering the sole array of possible phenotypes, since there exists a translation of this process into allele dynamics, even though it might be difficult or intractable to determine. At this level one answers the worry that reasoning about phenotypes, as in behavioral ecology, is often mere speculation given our ignorance of genetic makeups.

A second level is the adaptationist methodology, strictly speaking. The isomorphism connects dynamics of alleles to an optimizing program: to this extent, it not only provides grounds for addressing evolution at the phenotypic level with guarantees of a sufficient allelic underpinning, but it also allows one to use a reasoning parallel to optimizing methodology as used in economics, i.e., considering maximization of some value as the final state reached by the evolution of the phenotype.

Therefore, the equivalence between selection and optimization justifies the optimality methodology of behavioral ecologists, even if mathematical population genetics cannot prove that selection always maximizes fitness whatever the ecological and genetic circumstances. The support for this methodology cannot be demonstrated within population genetics as the science of the process of selective dynamics, but only through a general equivalence thesis between population genetics as a dynamics and an economic scheme centered on optimization. In economics, assuming the transitivity of preferences as the mark of rationality supports the possibility that agents maximize their utility, and such computation explains the choices; it is not an empirical claim that all agents behave rationally (i.e., maximize their utility function), but it means that rational deliberations are intrinsically directed toward maximized utilities. Analogously, in our framework, since the dynamics of selection can be turned into an economy whose agents choose phenotypes, natural selection is equally directed toward some maximum. Notice that this breaks the analogy between FD and fitness landscapes introduced before. Actually, landscape modeling is rather analogous to physics, in the sense that fitness function is construed exactly as potential functions in physics, and fits 
the same requisites, as Sewall Wright recognized. The landscape search for fitness extremes parallels the physical search for maximization of potential energy function as a heuristic for finding the final state of systems. But in the framework of FD, optimization rather parallels the maximization of utility in economics, thereby pertaining to another kind of methodology. One should pay attention to this switch in scientific models in order to understand what justifies optimality modeling and concepts in evolutionary theory.

To this extent we do not claim that selection will produce optimal traits everywhere, but that under basic conditions, defined above by the formal assumptions of FD, selection naturally optimizes, and on this basis, we can legitimately consider that optimality models are the default for evolutionary hypotheses. FD establishes that a trend toward optimizing is built into the very structure of selection, which may be phrased in terms of optimization even if in real life traits and phenotypes are not always optimized. It does not, then, justify "empirical adaptationism" (Godfrey-Smith 1998), i.e., the claim that many of the traits are indeed adaptations. If, in this world, conditions often do not satisfy the FD assumptions-viz., the strategic equivalence assumption-and some other requisites for intergenic selection, then we may not see so much adaptation. But "methodological adaptationism" (sensu Godfrey-Smith 1998; see also Mayr 1983), viz., the form of reasoning that starts with what should be optimal and makes a comparison with data, asking what prevents optimality being reached, correctly relies on the idea that the allelic dynamics of selection can be seen as an optimizing program run by organisms. As soon as conditions for natural selection are fulfilled (i.e., variation, heritability, and correlation between heritable variants and reproductive success; see Lewontin 1970), selection is acting, and we should expect optimality, and must check what were the non-basic conditions accounting for the lack of optimality.

In this perspective, the adaptationism controversy is reduced to a question about the kinds of reasons why optima are not reached by evolution. Many reasons have been studied, especially regarding the developmental constraints that preclude variation from providing the adequate variants for natural selection to optimize (Gould and Lewontin 1978; Maynard Smith et al. 1985; Lauder 1996). In the FD framework this can be translated into questions regarding the origins of the strategy set that is processed by the optimization program. But another class of reasons concerns the trade-offs that must be respected by the optimization program, and result in limits to optimization, viz., these suboptimal cases considered by the two last clauses of FD.

Trade-offs are themselves of two sorts. First, given that fitness is a compound magnitude, made up of survival and reproduction, one reason for the difficulty in reaching optimality relates to the constraints on the composition of fitness. There may be some trade-offs between survival and reproductive values. One example is cottonwood tree branches (Endler 1986): these branches are very fragile and thin, which seems to decrease the survival chances of the tree itself. Yet they actually improve the number of offspring by enhancing seed dispersal (less-fragile branches of variant cottonwood trees break less easily and therefore retain their seeds). Here, the reproduction component of fitness leads to the reproducing optimality of a trait at the expense of the robustness and plausible survival of the whole organism. A generalized instance of this situation is what the "life-history traits" research program (Stearns 1992) investigates, viz., the trade-offs between investments at various moments of the life cycle.

A second kind of trade-offs concerns the phenotypic traits themselves, as they are different traits carried by the same organism. These trade-offs may hold between selective pressures (e.g., a selective pressure to display few colors in order to avoid predators) and a sexual selection pressure that stems from the preference of females for conspicuous colors. Or they may hold between the traits themselves: if selection optimizes the speed race, regarding the predators as selective pressure, it also raises the metabolic costs, paid by other traits, and this often happens in a nonlinear way; which means that the genuine optimum is a trade-off between selection for speed and metabolic cost paid by other organs. Methodological adaptationism is always trade-off adaptationism.

Trade-off adaptationism concerns not only the issue of adaptation/optimality, but also the evolutionary role of the organisms that have the adaptations, hence it will also be considered in the next section. However, before ending this section I want to emphasize the specificity of the pluralistic framework I use here regarding the question of optimization. As indicated before, the formal grounds for believing that evolution optimizes something in organisms, which intuitively is implicit in the idea of adapted organisms, was Fisher's fundamental theorem. However, it is well known - and was precisely a motivation for FD - that population genetics does not support an idea of optimization. As stated above, it is not true that the mean fitness of a population is always increasing. But recent interpretations of the FTNS (Ewens 1989; Edwards 1994, after Price 1972) have shown that the meaning of the equality of additive genetic variance and change in fitness concerns exclusively the change in fitness directly caused by natural selection. As was visible in the first sentence of Fisher's book-"Natural selection is not evolution"-the FTNS concerns only natural selection and not evolution in general. It might be that other circumstances in evolution induce a decrease in mean fitness that is higher than the 
increase induced by selection, so in many cases, seen empirically or investigated in sophisticated population genetics models such as the ones mentioned above, evolution results in a decrease of a population's mean fitness. Fisher called those circumstances opposing the increase in fitness "deterioration of environment." But what he called environment can be either the external environment of organisms, or the genetic environment of alleles, which precisely results in the change of mean fitness of the alleles, thereby countering the effect of selection. This is exactly the case of frequency-dependent selection as mentioned in the beginning, which supposedly contradicted the FTNS.

So the updated meaning of the FTNS is that there is an intrinsic connection between natural selection and a trend toward maximization. It would in a way justify optimality reasoning in evolutionary theory. Using the FD framework thereby does not contradict the true insight of the FTNS. As highlighted above, on the basis of the FD one can only predict a trend toward optimization as soon as there is natural selection, but cannot expect optimization-maximization of inclusive fitness-in all cases. What Fisher saw as deterioration of the environment is, in the case of FD, understood as the conditions that would prevent the four clauses to hold, i.e., the negation of the assumptions of the FD isomorphism. The other similarity between FTNS and FD as supporting a justification for optimality reasoning is that both are established purely analytically. FD rests on the Price equation, which is an analytical truth linking $p$ score and fitness (Frank 1997), and FTNS is analytically derived from the connection between fitness (or "reproductive value"-Fisher) and additive genetic variance. Interestingly, the simplest derivation of FTNS is precisely done via the Price equation, by considering fitness as the phenotypic trait under focus (Price 1972). Therefore, the framework we consider here is derived wholly from what one could call the mathematics of natural selection. It is very plausible that the Price equation, FTNS, and FD are the same set of analytical truths about adaptation, selection, and, ultimately, design expressed in various ways, and possibly with different limiting assumptions, but investigating this is beyond the scope of this article.

However, the character proper of FD is that it ties the level of allele dynamics to the level of organisms. Therefore, although the FTNS establishes an optimality trend through natural selection, it remains at the level of alleles-or at least, it is hard to see the connection with organisms ${ }^{12}$ whereas FD provides a link between evolution as a process

\footnotetext{
${ }^{12}$ In the first formulations of the FTNS, Fisher spoke of the mean fitness of organisms, and indeed the integrity and design of organisms is in general crucially addressed by his theory; see, e.g., the evolution of dominance as it is understood in Fisher (1932).
}

understood by population genetics - the heart of the MS and organisms transforming through evolution, which in fact was Darwin's initial view of evolution.

I now turn to the second issue, organisms versus genes.

\section{Organisms and Genes as Perspectives on Evolution by Natural Selection}

I have shown that the pluralist framework defined by FD allows us to clarify the issue of adaptationism, the first axis of controversies about the MS. The second question is whether it also entitles us to reconcile the perspectives of genes and organisms, viz., to mediate between the view that evolution fundamentally happens at the level of genes (so that organisms are a mere epiphenomenal reflection of what happens there), and the view advocated by WestEberhard (2003), Walsh (2010), and others according to which genes are just a shadow of the activities of organisms, which produce evolution. I will successively address the questions whether within this pluralism MS can include organisms as "explanantia," and whether the facts of organisms indeed remain outside the explanatory scope of MS, as its critics claim.

\section{Organisms as Explanantia in the Modern Synthesis}

Population genetics formally corresponds to a view of individual organisms as agents that choose their phenotypes in order to maximize their inclusive fitness. Thereby, the organisms are included in the explanation of trait frequencies as "maximizing agents"; given that all of the alleles' dynamics can in principle be phrased in terms of maximizing organisms' activities, clearly the theory does not exclude organisms from its explanatory apparatus, nor does it reduce them to allelic processes.

The strength of this pluralism is to show that the understanding of the process of selection in terms of allele dynamics as elaborated since Fisher and Wright does not preclude considering organisms as agents in evolution. On the contrary, it decouples the idea of selection from the idea of adaptations as a kind of traits of organisms: for a trait to be an adaptation means to be an output of an optimizing program run by organisms; at the same time it also means the occurrence of a dynamics driven by selection at an allelic level. And because of this decoupling, such pluralism shows how these two notions are correlated, viz., through the isomorphism demonstrated in the FD and the subsequent clauses. It establishes that organisms and genes correspond to two specific takes on the process of natural selection that pertain to two explanatory schemes, respectively: one which is rather statistical-mechanical, the other which is rather economic. In this regard, genes and 
organisms should not be seen as levels, and their relation not reduced to the issue of the proper levels of selection (even if this is a question in its own right). Organisms and genes determine two explanatory schemes rather than two levels (because the idea of levels assumes some homogeneity, viz., the existence of a scale that includes all levels).

Considered in FD's pluralist framework, the MS is therefore not to be understood as expelling organisms from the explanatory core of the theory. Even if it rests on population genetics as the science of the process of selection, this does not downplay the role of organisms to mere vehicles for their genes (according to Dawkins' famous phrase), because besides selection as a process, an economic scheme could see selection as a program, considering organisms as agents in this program; and the two schemes are formally equivalent. Equivalence is not reduction. If one follows Ariew (2008) in considering that the MS changed the explanandum of evolution considerably—switching from Darwin's view of evolution as a transformation of organisms to a population-genetic view of evolution as gene dynamics - then one must consider that FD offers a pluralism that reconciles these two views. Therefore, advocating the explanatory role of organisms in evolution does not necessary mean a critique of the foundational role of population genetics in our knowledge of evolution.

\section{Organisms Explained: The Kantian Framework}

One way to address the "explanatory sufficiency" of the MS to capture organisms themselves is to start from the Kantian view of organisms, which is by and large the view developmental biologists have been sharing since the times of von Baer (Gilbert and Sarkar 2000, p. 3). Kant explained the design of organisms in terms of what he called "natural purpose." An entity showing design (Kant [1790]1987 §62, p. 364) is such that we cannot think of it without stating a "concept" at the source of its possibility. This is how Kant captures the problem raised by complex design and holistic integration as compared to chance (a problem addressed by Paley and others in theological terms): the concept at the source of the watch's production explains why, against the odds, there was a watch rather than some aggregate of wheels. Entities whose form and nature cannot be understood unless one presupposes that a concept was at the source of their possibility are said to be purposive. ${ }^{13}$

The notion of purposiveness is that even if contingent, those entities have some lawlikeness of their own when taken as living entities (Huneman 2014). Living entities are

\footnotetext{
13 Kant's Critique of Judgment ([1790]1987), §VIII, XLVIII, p. 192. See recent papers by Walsh $(2010,2012)$ to get a modern, scientifically informed, elaboration of such view.
}

contingent in the sense that it makes no difference for nature itself whether they are alive or not, or whether development leads to a monster or to a viable organism. But since the life sciences make these distinctions, and aim to capture some rules of this viability, we have to assume some necessity that guides development and rules the functioning of living systems in order to account for it (Ginsborg 2001; Huneman 2006). Purposiveness means therefore this "lawlikeness of the contingent as such." ${ }^{14}$ Such lawlikeness stems from our project of understanding life as such, and then does not have the same status as mere laws of nature, which, according to Kant, are at the basis of nature and of any knowledge in general. ${ }^{15}$ Contemporary philosophers such as Ratcliffe (2000) or McLaughlin (2001) have updated this Kantian position in philosophy of biology. ${ }^{16}$ Lewens (2007) spoke of "Kantian projectivism" in order to say that the concepts specifying purposiveness in organisms (above all, functions) stem from our scientific project.

Biological lawlikeness involves a specific relationship between the parts of a system and the system as a whole. Kantian purposiveness thus emphasizes the relationships between parts and whole instead of the means-ends relationships (with various connotations of utility) that long ago were the province of physiology and anatomy (Cunningham 2000), as when Galen investigated the "utilities" of various parts of organisms. Here, the main idea of purposiveness is the acknowledgement that parts have to be understood with reference to these wholes of which they are parts. ${ }^{17}$ Kant says that the idea of the organic whole is present as a principle of knowledge, ${ }^{18}$ meaning that it is not

\footnotetext{
${ }^{14}$ Kant ([1965] 2000), p. 20.

15 Kant makes it very clear that the presupposition of purposiveness allows the knowledge of living nature, so that if suppressed, it would leave intact the possibility of a physical science. It follows for instance from this: "They [those who study the structure of organisms] appeal to it just as they appeal to the principle of universal natural science-viz., that nothing happens by chance. Indeed they can no more give up upon that teleological principle than they can this universal principle. For just as abandoning this physical principle would lead them without any experience whatsoever, so abandoning that teleological principle would lead them without anything for guidance in observing the kind of natural things that have been once thought teleological, under the concept of natural purposes" ([1790]1987, §66, p. 376).

16 In another sense, Walsh (2006) explicitly connects an EvoDevo view of evolution to Kant's idea of organisms.

${ }^{17}$ It is only after this characterization of natural purpose in terms of parts and wholes that Kant recaptures the ancient meaning of purposiveness in terms of means and ends, a meaning usually found in the old "maxim of physiologists" (i.e., "The presupposition that all, in the animal, has its utility"; Kant ([1787]1998), p. A688/B716.

18 "The idea of the whole determines the form and the binding of all the parts: not as a cause, since it would be a product of art-but as a principle of knowledge [Erkenntnisgrund] of the systematic unity of the binding of all the manifold contained in the given matter for the one who has to judge" ([1790]1987, §65, p. 373).
} 
the principle of their making (otherwise we would be committed to the idea of a divine designer) but the very condition of our understanding of organs and traits as parts of an organism, ${ }^{19}$ i.e., as involved in the development and functioning of a living entity. Organisms are therefore "natural purposes" in the sense that we can only account for them if we presuppose this idea of a whole. "Natural purposes" are captured by two criteria:

[1] In such a product of nature each part, at the same time as it exists throughout all the others, is thought as existing with respect to the other parts and the whole, namely as instrument (organ). [2] That is nevertheless not enough (because it could be merely an instrument of art, and represented as possible only as a purpose in general); the part is thought of as an organ producing the other parts (and consequently each part as producing the others reciprocally). ([1790]1987, §65, p. 373; italics added)

Criterion (1) captures the general idea of purposiveness: each part has to be understood (and is what it is) because of its relation to the whole. There is an equivocation between technical and natural systems, both fulfilling the "design" criterion; this also allows one to think of organisms as akin to artifacts, in order to capture some of their complex adaptations (Lewens 2004). So the design criterion exactly captures the idea of design as displayed by organisms as well as technical complex objects - a set of contrivances which makes a system functionally likely to fulfill some end. ${ }^{20}$

\footnotetext{
19 "This principle does not pertain to how such things are possible themselves through this kind of production (things considered themselves as phenomena) but pertains only to the way our understanding is able to judge them" ([1790]1987, §77, p. 408).

${ }^{20}$ When compared to current thinking in developmental and evolutionary theory, the "design criterion" raises an important objection. Modularity is indeed crucial in development: developmentalists identify various "developmental modules" (Winther 2001) such as the ectoderm or the GRN of Endo 16 in the sea urchin, to the extent that those are coherent units (sometimes transitory) that function in relative isolation. (Physiological, morphological, and developmental modules differ, but they satisfy a common idea of modularity.) Modularity is also crucial to evolution because it facilitates it-without it, very few variations would be likely to increase fitness, since most would alter the overall functionality (Wagner and Altenberg 1996). It first seems that this design criterion runs counter to the acknowledgement of the role of modularity in development and evolution. However, what is important in the design criterion is that the parts must be understood in relation to the whole, which means that they can be conceived of as fulfilling a functional role. In this sense the ectoderm has a crucial functional role in development, just as a physiological module such as the wing has a functional role in vital activities. It does not mean that the actual functioning should depend on the whole, only the role. The module cannot be understood without the whole, but can function by itself; likewise the whole can function without the module, though less well, and this difference of degree is of course a matter of empirical investigation on a case-by-case basis.
}

Criterion (2) that I call, epigeneticity, distinguishes organisms from artifacts because their design, in the sense of an arrangement of the parts according to an idea of the whole, is not achieved by some external agent who considers the idea of the whole as some sort of building plan. ${ }^{21}$ Technique happens when the idea of the whole acts as a "cause" through human agents; but when organisms occur this idea of the whole exists only as a "principle of knowledge" (Kant [1790]1987, §65, p. 373), and thus allows us to understand development and functioning. Therefore it is the parts themselves that perform the process of building organisms, hence they produce themselves, and they have to be considered as acting according to an idea of the whole. ${ }^{22}$ In this sense, the epigeneticity criterion accounts for organisms as entities with an epigenetic character: they are constantly producing themselves during embryogenesis and through their normal functioning. Organized beings are beings that "self-organize," said Kant, ${ }^{23}$ and this is why they can be conceived of as "natural purposes." And whereas the first criterion involves a causal relation no stronger than just a simple relation of mutual dependence (like wheels and gears whose forms have to be adjusted in order to make a mechanism functional but are not produced one by the other and result from a common cause), the epigeneticity criterion is causal in the sense that causation also means production (involving locality, contiguity, conserved quantities in time, and so on). These two senses of causation are prima facie (and may remain irreducibly) distinct, since according to Hall (2004) dependence and production fulfill two distinct sets of requisites (especially regarding locality). ${ }^{24}$

\section{Accounting for the Design Criterion}

That the economic scheme of selection is an optimization program now accounts for the fact that organisms will indeed satisfy the design criterion, viz., be integrated

\footnotetext{
21 "Thus, concerning a body that has to be judged as a natural purpose in itself and according to its internal possibility, it is required that the parts of it produce themselves [hervorbringen] together, one from the other, in their form as much as in their binding, reciprocally, and from this causation on, produce a whole" (Kant [1790]1987, §65, p. 373).

${ }^{22}$ The concept of "original organization" in the context of embryology instantiates this idea of the whole as a principle of knowledge. See Huneman (2007) for the connection between embryology, epigenesis, and Kant's view of regulative principles.

23 If something meets the criteria of a natural purpose, then it "is both an organized and a self-organizing being" (Kant [1790]1987 §65, p. 374). Kauffmann (1993) quotes this as related to his own work. Theoreticians of "autopoiesis" like Varela also invoke this Kantian statement.

${ }^{24}$ Philosophical accounts of causation in terms of counterfactuals capture rather the former sense, whereas accounts in terms of process (Salmon 1984; Dowe 1992) capture rather the second one.
} 
wholes. According to FD, given that the maximand of the $g$ function turns out to be inclusive fitness, natural selection makes it impossible to have non-integrated wholes as sets of traits. Suppose that a whole organism is not integrated. As a result, it will be less functional than other, more integrated, variants-because "to function physiologically" presupposes the ability to perform several functions at the same time, and the more one minimizes antagonisms between parts doing various functions, the more one optimizes the survival chances and, accordingly, the chances of reproduction. Therefore, as maximizing agents, organisms will necessarily optimize their holistic design. Thus, a first answer to the organismal criticism of MS would be that the economic scheme of selection-optimization leads to entities that are holistically composed, because their traits, when selected, should also be cohesive to the extent that cohesion with other traits is involved in the definition of maximizing fitness.

Actually, this design closely follows from the "trade-off adaptationism" emphasized above. In this sense, the "designedness" of organisms as functional wholes is guaranteed by FD, but entails a cost to the value of particular traits, because they are contrived by the other traits. In this context, the traits whose value is optimized by selection are constrained by the systematicity of organisms, which also derives from selection. Here, these constraints are understood in the same economic framework as all the traits of the agents are understood. Thereby the design of an organism can be seen as explained by selection, and in the end it results from the dynamics of alleles.

\section{Making Sense of the Epigeneticity Criterion in the Current Context}

The epigeneticity criterion is fulfilled when Kant's notion of circular self-organization is instantiated in a system. Recent knowledge of developmental processes-actually, much more recent than Kant's theories-has shown such self-organizing patterns in living systems. At first sight, cells indeed produce cells through mitosis, so that organismal parts effectively cause other parts. But even if all cells have the same genome, hence the same possibilities to express cell phenotypes, emerging cells have the adequate cell type according to their position in the organism. A closer understanding of this process shows it fits the Kantian scheme: the cell types of the cells produced by cells are conditioned by their location and neighborhood within the organism-thus we have to assume the overall functionality of the organism in order to address and empirically investigate this plurality of cell types, together with the division of labor it comes from.

A standard developmental model for pattern formation, according to which the cells are informed about which type they should express through their spatial position, is Wolpert's (1994) "French flag model." What "tells" cells their fate is the gradient of a substrate, the "morphogen," expressed by all the surrounding cells. Here, clearly, cells cause other cells to adopt their types because they produce the morphogen gradient. Cells have a causal role in producing other cells_-and in producing ultimately the wholly developed organism-through a production scheme that relies on local conditions but results in the organized set of cell types.

One could object that such self-production by cells is just the expression of the genetic program encoded in the nucleus. However, this overlooks the real functioning of genes within organisms: activation states of the genes are not produced by themselves, but by cellular and intracellular environments of genes; e.g., the methylation state of each allele depends upon this environment and is inherited by the daughter cell after mitosis (Jablonka and Lamb 2005). This contributes to accounting for cell differentiation, which entails that not all the genes of a cell are activated in different parts of an organism. Moreover, the transduction of genes (RNA) into proteins is not enough to account for functional proteins, and other factors (physicochemical components of the normal physiological medium and other macromolecular structures) are involved in the three-dimensional folding of proteins. Actually, it is this folded protein and not the sequence given by the DNA (underdetermining the structure) that is functional in the organism. In turn, the kind of three-dimensional protein thus determined may act on the activation and regulation of the genome. Summing up: from the perspective of cells an organism seems to be continuously producing itself through those causal loops between DNA, RNA, and epigenetic, intra- and extra-cellular factors and environments. $^{25}$

The MS, interpreted through the FD framework, shows that the design of organisms can be understood in terms of natural selection driving an allele dynamics. The question of the relevance of MS to the understanding of organisms finally boils down to the question of whether the epigeneticity criterion, that is, the self-organizing patterns especially shown by developmental processes, can in turn be understood through the selective dynamics of alleles, likely to be translated in optimization schemes.

\footnotetext{
25 The idea of a developmental cycle, advocated by Developmental Systems Theory (DST; Griffiths and Gray 1994) as the unit of evolution against the MS, could be derived from those considerations, although it is a more demanding hypothesis. I do not enter into this controversy, but only notice that DST is one possible philosophical position on the basis of those findings (See Huneman 2013 for a discussion of DST along similar lines).
} 
Self-Organization, Epigenetics, and Gene Networks

To decide to what extent MS through FD captures this selforganizing aspect of organisms, one must determine what would be an empirical test. Here is a way to tackle this problem.

Development involves circular processes that, as we have seen, determine the self-organizing cohesiveness of organisms. Genes are an essential part of these processes, but insufficient, as has been extensively argued (e.g., Neumann-Held 2001; Moss 2003; Jablonka and Raz 2009; etc.). And genes are also the units in this selective dynamics that is isomorphic to organisms' optimizing processes. Therefore, the more important and relevant genes are within the former circular processes, the more the self-organizing aspect (and thereby the epigeneticity criterion) can be understood through the selective dynamics. Having a measure of the relative weight of the genes involved in such processes could indicate to what extent the MS can account for this other side-the epigeneticity criterion-of organisms.

Such an assessment is possible if one considers the molecular biology of these processes. A crucial insight in cell differentiation was given through the concept of gene regulatory networks (GRN), initiated by Davidson (1986). GRNs are networks of genes modeling the relationships of inhibition, repression, and activation that, depending on the present state of the cell and its surroundings, lead to silencing or expressing genes in the genome. The topology of the network represents the signaling, inducing, repressing, and expressing pathways, whereas its dynamics captures the variation in quantitative variables (amount of gene products, etc.) according to some equations (e.g., Michaelis-Menten). The GRNs are responsible for the cell specification, "the acquisition of a given regulatory state, as the sum of the activities of the transcription factors expressed in the cell nuclei" (Oliveri et al. 2008), which happens during life in general. They have proved to be crucially involved in the formation of patterns during development (Davidson et al. 2003; Levine and Davidson 2005), since pattern formation rests on specified cell lineages. ${ }^{26}$ At each stage of development, according to the conditioning relations within the network, they process an input-for example a maternal protein at the inception of mesoderm formation-into a phenotypic profile of the cell, ${ }^{27}$ which influences other cells' behaviors. GRNs are

\footnotetext{
${ }^{26}$ For example, Oliveri et al. (2008) have construed the complete GRN of skeletogenic micromere lineage in sea urchins (Strongylocentrotus purpuratus), i.e., the cell lineage that during sea urchin ontogenesis gives rise to the skeletogenic mesenchyme, and develops after the fourth cleavage up to the gastrulation stage.

27 "Spatial cues are always required in order to trigger specification in development. These spatial cues sometimes consist of localized
}

flexible, and change along the organism's life cycle-the GRNs involved in development are not necessarily the GRNs involved in physiological regulation of cell behavior.

Gene regulatory networks as networks of gene activities do not exclude non-genic factors such as epigenetic ones. The complete interaction loops involving gene activities also comprise proteins and transcripts, which are both modifying and modified specific gene activities. So cells developmentally and physiologically produce their own states and other cells, in response to environmental conditions, on the basis of such a set of conditioning interactions. To this extent the GRN can, strictly speaking, be seen as a projection of the total regulative network (involving proteins and transcripts) onto the sole level of gene activities ${ }^{28}$ (see Fig. 3). GRNs are our way of modeling the inter-level interactions that control cell development and behavior in accordance with the cell neighborhood, and the overall organism in general. Hence they are involved in the causal loops through which cells build other cells, and construct organisms; reciprocally, organisms determine (through intermediary levels) the nature and state of cells.

Is it possible to assess the relative weight of genes in these complete processes? It would seem it is. Actually, one can ask what is the relative effect of changing genes and gene states with regard to changing the other factors in the overall network. After the investigation of the GRN involved in specifying the skeletogenic micromere lineage in the sea urchin (Oliveri et al. 2008) was completed, it turned out that the genome itself was the most important

\section{Footnote 27 continued}

maternal regulatory factors that are distributed to particular cells with the egg cytoplasm, and are partitioned during cleavage. Alternatively they can also consist of signaling ligands produced by other cells, in consequence of their own prior state of specification. Ultimately, these spatial cues affect the course of events in development by causing the activation (or repression), in a certain region of the embryo, of particular genes encoding transcription factors. Through this process, new, more refined, domains of specification are created, and the complexity of the embryo increases" (Revilla-I-Domingo and Davidson 2003, p. 695).

28 "The directed edges in GRNs correspond to causal influences between gene activities. These could include regulation of transcription by transcription factors, but also less intuitive causal effects between genes involving signal transduction or metabolism. It is of uttermost importance to realize that when inferring GRNs from geneexpression data alone, the metabolites and proteins act as hidden variables. These variables mediate communication between genes, but since they are not included explicitly in the GRNs, only their effects appear as edges between the observed variables, i.e., gene activities. GRNs thus describe communication between genes implicitly including all regulatory processes inside and therefore give a complete description of cellular regulation projected on the gene activities.... Influences between gene-activities, without explicitly taking account for the proteins and metabolites, result from a projection of all regulatory processes on the 'gene space"" (de la Fuente 2009, p. 3). 
Fig. 3 a Network of protein, metabolic, and gene interactions. b Graph of GRN as a projection of the network (shown in a) on gene space (after de la Fuente 2009, p. 8)

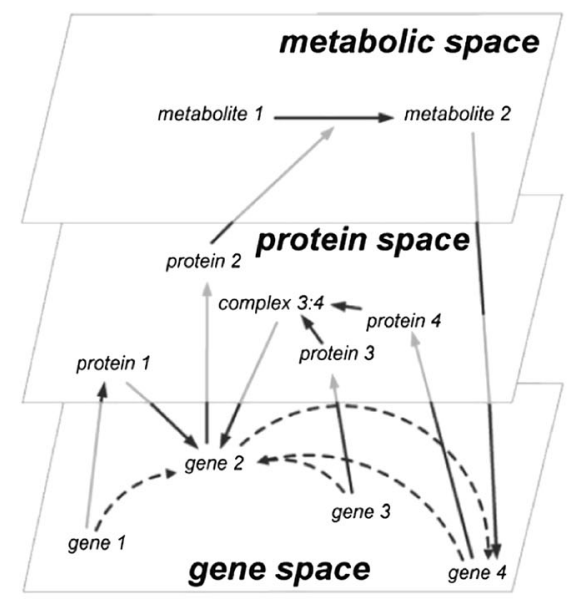

A

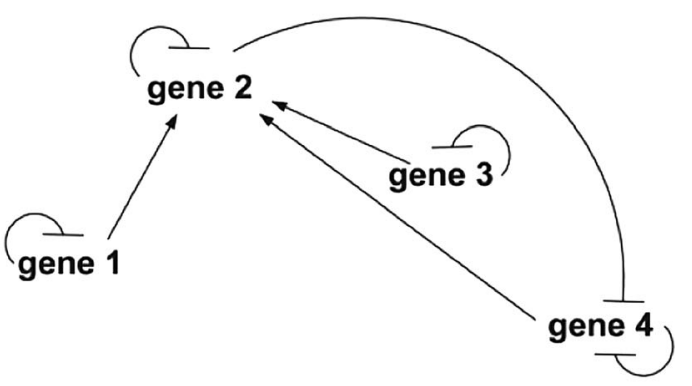

B causal factor in the final GRN performance, meaning that changing the transcripts and proteins (environmental inputs of the GRNs) on average does not change the outcome much. Researchers (Hood 2008) asked whether the GRNs involved in physiology rather than development will share the same property, or will offer a more important role to non-genomic factors.

Gene regulatory networks are projections of sets of relations between three spaces (Fig. 3a); hence the question of relative weights can be interpreted topologically. From this viewpoint, not all possible patterns of projections are equal. Some of them ("type 1") are such that many different interactions with proteins are projected upon the same gene activity, i.e., the projection onto the gene space is quite resistant to what happens within other spaces (that is, it does not change a lot when one wiggles the overall network in other spaces). This robustness means that the major causal effect is due to gene changes, because differences made to other products' activities do not make much difference to the overall result. Another class of projections ("type 2") is such that the set of proteins and metabolic interactions defined by the small changes in a given one has many projected images on the gene space instead of just one.

In type 1 , genes are much more what counts because information about proteins and metabolites is not as important to understanding the network, since they can be abstracted away when the functioning of the GRN is analyzed. In type 2, all spaces are important. These types of projections correspond to two kinds of structures of overall networks, and the question of the relative weight of genes in self-organizing processes (i.e., the question of the accountability of the "epigeneticity criterion" in terms of selective dynamics) is whether most of them are of type 1 or type 2 . Therefore, in the set of type 1 networks, most of the variation between phenotypes so regulated is due to gene differences, whereas this is obviously not the case in the set of type 2 networks.

Formulated in these terms, Oliveri et al. (2008) found that the structure of the whole network of interactions (across the three spaces) for the skeletogenic micromere lineage in the development of sea urchins is of type 1 , since tweaking proteins or transcripts does not much modify the behavior of the network - contrary to tweaking the genesso that the projections of different epigenetic parts onto the genetic space are equal.

Thus, developmental GRNs that have been investigated already are more often of type 1; if all GRNs (including physiological ones) turn out to be mostly of this type, this would empirically support the claim that epigenetic processes are not as crucial as gene dynamics for understanding the evolution of organismic systematicity. This would of course not mean that as such they are superfluous, but simply that their differences do not make much of an impact as compared to the effect gene differences have: the latter will much more strongly determine change in the phenotypic outcome of the GRN in the cell. In this case the causal loops by which genes, cells, extracellular products, and organisms are intertwined in producing design could be abstracted away, the mere understanding of genomes and their evolution being explanatorily relevant. Of course, deciding such issues will not settle the question, since many other arguments, much closer to EvoDevo-style investigations, call for the importance of epigenetics in understanding the fact of organisms and their role in evolution, especially when it comes to non-genetic inheritance (Helantera and Uller 2010; Danchin et al. 2011). Yet, formally determining what types 1 and 2 are, and finding whether actual GRNs are mostly of type 1 or type 2 , would provide an example of how to empirically settle the question of the explanatory irreducibility of the self-organizing patterns of organisms regarding MS. 


\section{Conclusion}

Formal Darwinism has demonstrated that the MS provides a legitimate account of the systematicity in organisms through its view of Darwinian explanatory pluralism having two sides, economics-like and physics-like, i.e., optimization and allelic dynamics. At the same time, it legitimates optimality views in evolution as these can be taken as the default in a perspective where organisms are seen as agents. Rather than trying to ground optimality through population genetics via the FTNS as attempted in the past, FD justifies optimality by reinterpreting the whole physical framework of population genetics in terms of economics.

This does not mean that selection makes everything optimal, or that an organismal biology can be wholly derived from the sub-organismic biology of gene frequencies. Rather, this pluralism shows how optimization as a default is embedded in selection, and how organismic systematicity to some extent derives from the inclusivefitness driven economy of maximizing agents. Lastly, by examining the relative weights of genetic and non-genetic components of GRN after the example of developmental GRNs, I suggested that there are empirical ways to decide whether the properties captured by the "epigeneticity criterion," and which are definitive of organisms, can to some extent be accounted for in the MS framework.

\section{References}

Amundson R (2005) The changing role of the embryo in evolutionary thought. Cambridge University Press, Cambridge

Ariew A (2008) Population thinking. In: Ruse M (ed) Handbook of philosophy of biology. Oxford University Press, Oxford, pp 64-86

Bateson P (2005) The return of the whole organism. J Biosci 30:31-39

Brandon RN (1990) Adaptation and environment. Princeton University Press, Princeton

Cunningham A (2000) The pen and the sword: recovering the disciplinary identity of physiology and anatomy before 1800 . I, Old physiology - the pen. Stud Hist Philos Biol Biomed Sci C33:661-678

Danchin E, Charmantier A, Champagne FA, Mesoudi A, Pujol B, Blanchet S (2011) Beyond DNA: integrating inclusive inheritance into an extended theory of evolution. Nat Rev Gen $12: 475-486$

Davidson EH (1986) Gene activity in early development. Academic Press, Orlando

Davidson E, McClay D, Hood L (2003) Regulatory gene networks and the properties of the developmental process. Proc Natl Acad Sci USA 100:1475-1480

de la Fuente A (2009) What are gene regulatory networks? In: Das S, Caragea D, Welch S, Hsu WH (eds) Handbook of research on computational methodologies in gene regulatory networks. Medical Information Science Reference, Hershey, PA, pp 1-27

Dowe P (1992) Wesley Salmon's process theory of causality and the conserved quantity theory. Philos Sci 59:195-216
Edwards AWF (1994) The fundamental theorem of natural selection. Biol Rev Camb Philos Soc 69:443-474

Endler J (1986) Natural selection in the wild. Princeton University Press, Princeton

Ewens WJ (1989) An interpretation and proof of the fundamental theorem of natural selection. Theor Popul Biol 36:167-180

Fisher RA (1930) The genetical theory of natural selection. Clarendon, Oxford

Fisher RA (1932) The bearing of genetics on theories of evolution. Sci Prog 27:273-287

Frank SA (1997) The Price Equation, Fisher's fundamental theorem, kin selection, and causal analysis. Evolution 51:1712-1729

Frank SA, Slatkin M (1992) Fisher's fundamental theorem of natural selection. Trends Ecol Evol 7:92-95

Gardner A (2009) Adaptation as organism design. Biol Lett 5:861-864

Gilbert SF, Epel D (2009) Ecological developmental biology. Sinauer, Sunderland

Gilbert SF, Sarkar S (2000) Embracing complexity: organicism for the twenty-first century. Dev Dyn 219:1-9

Gilbert SF, Opitz G, Raff R (1996) Resynthesizing evolutionary and developmental biology. Dev Evol 173:357-372

Ginsborg H (2001) Kant on understanding organisms as natural purposes. In: Watkins E (ed) Kant and the sciences. Oxford University Press, Oxford, pp 231-259

Godfrey-Smith P (1998) Complexity and its function in mind and nature. Cambridge University Press, Cambridge

Gould SJ, Lewontin RC (1978) The spandrels of San Marco and the Panglossian paradigm: a critique of the adaptationist programme. Proc R Soc Lond B205:581-598

Grafen A (1984) Natural selection, kin selection and group selection. In: Krebs JR, Davies NB (eds) Behavioural ecology. Blackwell, Oxford, pp 62-84

Grafen A (2002) A first formal link between the Price equation and an optimisation program. J Theor Biol 217:75-91

Grafen A (2006) Optimisation of inclusive fitness. J Theor Biol 238:541-563

Grafen A (2007) The formal Darwinism project: a mid-term report. J Evol Biol 20:1243-1254

Griesemer J (2000) Development, culture, and the units of inheritance. Philos Sci 67:2348-2368

Griffiths PE (2001) Genetic information: a metaphor in search of a theory. Philos Sci 68:394-412

Griffiths PE, Gray R (1994) Developmental systems and evolutionary explanation. J Philos 91:277-304

Grossberg R, Strathmann R (2007) The evolution of multicellularity: A minor major transition? Annu Rev Ecol Evol Syst 38:621-654

Hall N (2004) Two concepts of cause. In: Collins J, Hall N, Paul LA (eds) Causation and counterfactuals. MIT Press, Cambridge, pp 225-276

Helantera EH, Uller T (2010) The Price equation and extended inheritance. Philos Theory Biol 2:1-17

Hood L (2008) Gene regulatory networks and embryonic specification. Proc Natl Acad Sci USA 105:5951-5952

Huneman P (2006) From comparative anatomy to the 'Adventures of reason.' Stud Hist Philos Biol Biomed Sci 37:649-674

Huneman P (2007) Reflexive judgment and embryology: Kant's shift between the First and the Third Critique. In: Huneman P (ed) Understanding purpose: Kant and the philosophy of biology. University of Rochester Press, Rochester, pp 75-100

Huneman P (2010) Assessing the prospects for a return of organisms in evolutionary biology. Hist Philos Life Sci 32:341-372

Huneman P (2013) Causal parity and externalisms: extensions in life and mind. Minds Mach 23(3):377-404

Huneman P (2014) Purposiveness, necessity, and contingency. In: Watkins E, Goy I (eds) Kant's philosophy of biology. de Gruyter, Berlin, pp 185-202 
Jablonka E, Lamb M (2005) Evolution in four dimensions. MIT Press, Cambridge

Jablonka E, Raz G (2009) Transgenerational epigenetic inheritance: prevalence, mechanisms, and implications for the study of heredity and evolution. Quart Rev Biol 84:131-176

Kant I ([1787] 1998) Critique of pure reason. Ed. \& trans. by Guyer P, Wood A. Cambridge University Press, Cambridge

Kant I ([1790]1987) Critique of judgment. Hackett, Indianapolis. German orig. (1913) Kant's gesammelte Schriften, Akademie Ausgabe, Bd 5, De Gruyter, Berlin

Kant I ([1965] 2000) First introduction to the Critique of the Power of Judgment. In Kant I, Critique of the Power of Judgement. Ed. \& trans. by Guyer P, Matthews E. Cambridge University Press, Cambridge, pp 3-51. German orig. (1965) Kant's Gsammelte Schriften, Akademie Ausgabe, Bd 20, De Gruyter, Berlin, pp 195-251

Kauffmann S (1993) Origins of order: Self-organization and selection in evolution. Oxford University Press, Oxford

Lauder GV (1996) The argument from design. In: Rose MR, Lauder GV (eds) Adaptation. Academic Press, San Diego, pp 55-91

Levine M, Davidson E (2005) Gene regulatory networks for development. Proc Natl Acad Sci USA 102:4936-4942

Lewens T (2004) Artefacts and organisms. MIT Press, Cambridge

Lewens T (2007) Functions. In: Matthen M, Stephens C (eds) The handbook of philosophy of biology. Elsevier, Amsterdam, pp 526-547

Lewontin RC (1970) The units of selection. Annu Rev Ecol Syst $1: 1-18$

Lewontin RC (1978) Adaptation. Sci Am 239:212-228

Lynch M (2007) The origins of genome architecture. Sinauer, Boston

Marshall CR, Orr HA, Patel NH (1999) Morphological innovation and developmental genetics. Proc Natl Acad Sci USA 96:9995-9996

Maynard Smith J, Burian RM, Kauffman SA et al (1985) Developmental constraints and evolution. Q Rev Biol 60:265-287

Maynard-Smith J (1982) Evolution and the theory of games. Oxford University Press, New York

Mayr E (1983) How to carry out the adaptationist program. Am Nat 121:324-334

Mayr E (1997) The objects of selection. Proc Natl Acad Sci USA 94:2091-2094

McLaughlin P (2001) What functions explain? Cambridge University Press, Cambridge

Moran P (1964) On the non-existence of adaptive topographies. Ann Hum Genet 27:383-393

Moss L (2003) What genes can't do. MIT Press, Cambridge

Neumann-Held E (2001) Let's talk about genes: the process molecular gene concept and its context. In: Oyama S, Griffiths
PE, Gray RD (eds) Cycles of contingency. MIT Press, Cambridge, pp 69-84

Odling-Smee J, Laland K, Feldman M (2003) Niche construction: the neglected process in evolution. Princeton University Press, Princeton

Oliveri P, Tu Q, Davidson E (2008) Global regulatory logic for specification of an embryonic cell lineage. Proc Natl Acad Sci USA 105:5955-5962

Orr HA, Coyne JA (1992) The genetics of adaptation revisited. Am Nat 140:725-742

Oyama S (1985) The ontogeny of information. Cambridge University Press, Cambridge

Price GR (1972) Fisher's fundamental theorem made clear. Ann Hum Genet 36:129-140

Ratcliffe M (2000) The function of functions. Stud Hist Philos Biol Biomed Sci 31:113-133

Revilla-I-Domingo R, Davidson E (2003) Developmental gene network analysis. Int J Dev Biol 47:695-703

Salmon WC (1984) Scientific explanation and the causal structure of the world. Cambridge University Press, Cambridge

Sober E (1998) Three differences between deliberation and evolution. In: Danielson P (ed) Modeling rationality, morality, and evolution. Oxford University Press, pp 408-422

Stearns SC (1992) The evolution of life histories. Oxford University Press, New York

Turner S (2001) The extended organism. Oxford University Press, New York

Wagner GP, Altenberg L (1996) Complex adaptations and the evolution of evolvability. Evolution 50:967-976

Walsh D (2006) Organisms as natural purposes: the contemporary evolutionary perspective. Stud Hist Philos Biol Biomed Sci 37:771-791

Walsh D (2010) Two neo-Darwinisms. Hist Philos Life Sci 32:317-339

Walsh D (2012) Mechanism, emergence, and miscibility: the autonomy of Evo-Devo. In: Huneman P (ed) Functions: selection and mechanisms. Springer, Dordrecht, pp 43-65

West-Eberhard MJ (2003) Developmental plasticity and evolution. Oxford University Press, Oxford

Williams GC (1992) Natural selection: levels, domains, and challenges. Oxford University Press, Oxford

Winther RG (2001) Varieties of modules: kinds, levels, origins, and behaviors. J Exp Zool (Mol Dev Evol) 291B:116-129

Wolpert L (1994) Positional information and pattern formation in development. Dev Genet 15(6):485-490

Yakubu Y (2013) The altruism paradox: a consequence of mistaken genetic modeling. Biol Theory 8:103-113 